\title{
Operation mode of electric business logistics based on the application of two-dimensional code technology
}

\author{
Jing Rao* \\ Department of Financial Management, Chongqing Youth Vocational \& Technical College, Chongqing 400712, PR China
}

Received: 1 November 2019 / Accepted: 28 January 2020

\begin{abstract}
In recent years, with the irresistible pace of e-commerce development, e-commerce logistics orders show blowout growth. For the logistics industry, it is both an opportunity and a difficult problem as there are many problems such as low technology content and poor distribution efficiency. Therefore, it is a challenge that needs to be tackled to integrate technology accurately with the operation mode of e-commerce logistics. In order to improve the effect of logistics operation, bring convenience to the management of business logistics, and ultimately realize the maximum profit of logistics enterprises, based on two-dimensional code technology, this study took the specific mode of logistics operation as the research subject, applied the corresponding twodimensional code generated to three different logistics processes, put forward specific optimization schemes, and carried out the analysis of the application results. The results showed that the intervention of two-dimensional code could protect users' privacy, prevent channel conflict, and realize logistics tracing of product information, thus effectively improve the logistics operation mode.
\end{abstract}

Keywords: Two-dimensional code / e-commerce / decoding / logistics tracing

\section{Introduction}

With the rapid development of economy and the popularization of Internet, e-commerce emerges as new industry. In e-commerce, merchants and customers do not need to deal face-to-face, but only need to place an order online. In order to complete the transaction, they need to deliver the goods to customers. Therefore, the rise of ecommerce has also led to the development of the express industry. In the traditional express logistics process, express documents are marked in clear text, so the customer's privacy information is very easy to leak, and it is difficult to track the logistics track in real time. With the emergence of two-dimensional code and wireless communication technology, the traditional logistics mode has changed, which makes the encryption and tracing of logistics information possible. Its basic principle is to upload the plaintext personal and cargo information to the system of the logistics company and generate the corresponding two-dimensional code after encryption, and the logistics track information can be uploaded or queried after connecting the system through the twodimensional code. Zhang et al. [1] encrypted personal information based on segment encryption method and stored the ciphertext in the two-dimensional code using

* Corresponding author: $r j$ jing@yeah.net
Logistics Information Privacy Protection System (LIPPS) of encrypted two-dimensional code and designed an authorization mechanism to decrypt the corresponding information and complete the operation of logistics business. In order to solve the problem of inefficiency and poor effect of credit authentication system in logistics process, Shan et al. [2] put encrypted data in credit authentication website into two-dimensional code generation system with MMS as carrier, identified two-dimensional code image through special identification equipment, and transmitted the result to two-dimensional code generation system through General Packet Radio Service (GPRS) wireless, and achieved credit authentication in the background of site. Xiao et al. [3] put forward an aquatic product quality and safety traceability system which combining wireless sensor network with the two-dimensional code technology, carried out experiment in actual cold chain, and found that the system could effectively realize the real-time monitoring and tracking of aquatic product cold chain and the two-dimensional code provides an effective means for the error correction and query of static and dynamic information query. Di et al. [4] proposed a two-dimensional code recognition technology for identifying labels on packages in automatic packet sorting system and found that the method was effective and had superior performance than other algorithms. Kalkan et al. [5] analyzed data matrix codes and two-dimensional codes. After printing the code, it was irradiated by X-ray to 


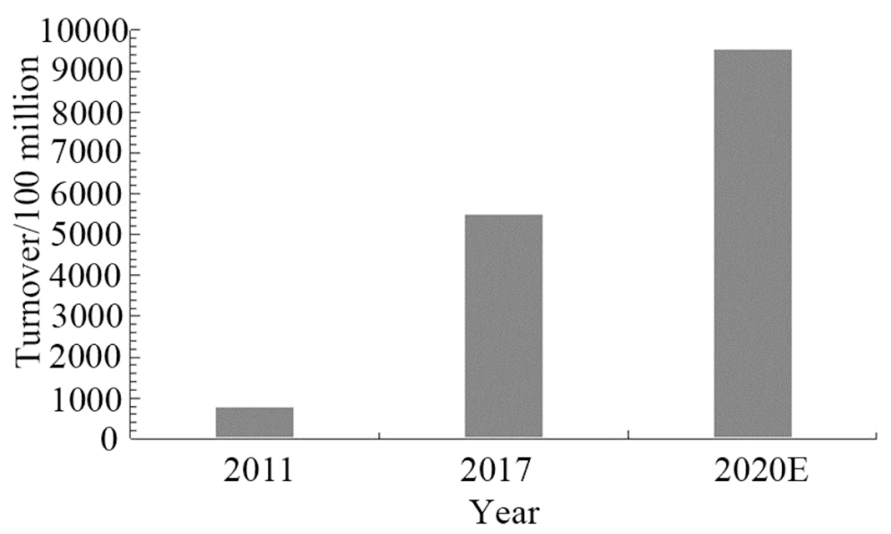

Fig. 1. Annual e-commerce turnover from 2011 to 2020 (predicted).

expose to an accelerated aging environment and immersed in water. All printed samples before and after irradiation and accelerated aging were read using laser and Charge Coupled Device (CCD)-based readers, and the minimum grating hue values for each printing technology and each printing ink were determined. To better apply the twodimensional technology in e-commerce logistics, based on the operation mode of e-commerce logistics, two-dimensional code was combined with logistics to solve problems including personal information protection, channel conflict and product information traceability in e-commerce logistics links, specific application schemes were put forward, and finally the application results of twodimensional code were analyzed.

\section{E-commerce logistics}

E-commerce is a new industry developed under the strong background of online shopping in the era of big data, which is a new growth point of China's economy [6]. E-commerce logistics [7] is the derivative industry of e-commerce and the last link of e-commerce realization. E-commerce logistics refers to setting up logistics transfer stations between buyers and sellers and warehousing and distributing goods, which can control all activities in the whole process after delivery of goods in real time. In addition to information services, almost all products traded by ecommerce are delivered by order fulfilled by e-commerce logistics [8]. At present, the order completed amount of ecommerce logistics is increasing, and a variety of new logistics enterprises emerge in endlessly. The development of the "Internet +" era will be a great opportunity for the integration of Internet and traditional products and the development of a new mode of "Internet + logistics". Figures 1 and 2 show the turnover of e-commerce and the completed business volume of e-commerce logistics in China from 2011 to 2020 (predicted) respectively.

\section{Two-dimensional code}

Two-dimensional code refers to two-dimensional barcode, which is a kind of barcode. It is a technology that first stores

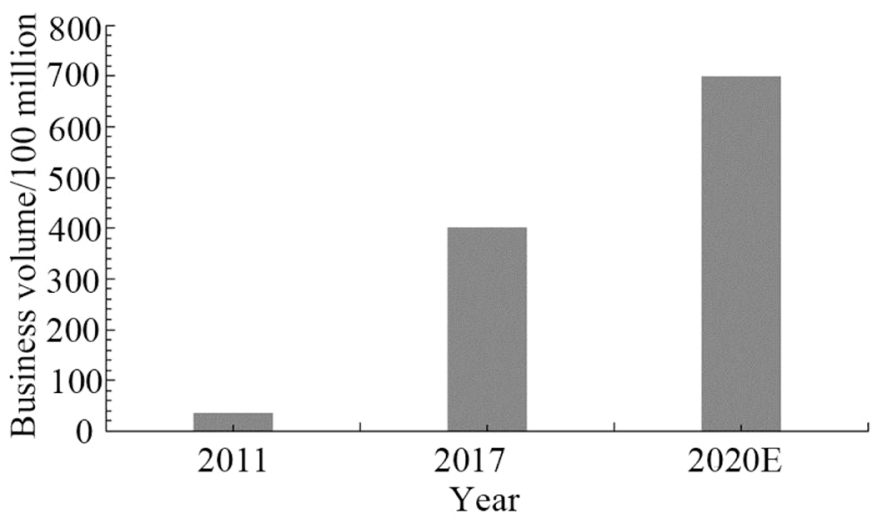

Fig. 2. Annual e-commerce logistics business volume from 2011 to 2020 (predicted).

information in barcode and then reads information through corresponding scanning equipment when reading [9]. In the two-dimensional plane of the two-dimensional code, there are many rectangles with different light and shade and different sizes arranged alternately. The various features of rectangles represent the information to be saved. Generally, two-dimensional code has advantages of large storage capacity, high recognition rate and high confidentiality, which can effectively improve the efficiency of information collection. According to implementation methods, there are stacked two-dimensional code and matrix twodimensional code, among which, matrix two-dimensional code [10] is relatively widely used.

\section{Two-dimensional code application scheme}

\subsection{Two-dimensional code based personal information protection scheme}

In order to record conveniently, the courier sheet often contains a large number of users' real personal information. Once this information is leaked, it may bring hidden dangers to users' personal safety. Therefore, protecting that information by two-dimensional code technology is a new mode of logistics operation.

\subsubsection{Secret key distribution}

As the work at the beginning and end of the e-commerce logistics process is the closest to the seller and buyer, it is necessary to know the details of users for normal work; however, each transit station only needs to know which distribution station the next station should be sent to. The secrete key distribution related to e-commerce goods among logistics personnel is shown in Table 1. Logistics personnel gets the secrete key which belongs to the scope of their authority and stores the secrete key in the terminal database $(i=1,2,3, \ldots, n-1)$. Because of the particularity of the duty of sending personnel at the terminal, it is necessary to decrypt the express delivery information first to get the delivery address and then to protect the address information by encrypting, in order to prevent accidental leaking of information in the process of distribution due to express storage and other reasons. 
Table 1. Distribution of secrete key for logistics personnel.

\begin{tabular}{llll}
\hline Logistics personnel identity & Business operation & Authorization level & Secrete key held \\
\hline Receiving personnel & Encrypt & 1 & Public key \\
& & 2 & Auth_i_pub Key \\
Transferring personnel & Decrypt & 3 & Private key \\
& & $\ldots$ & Auth_i_pri Key \\
Sending personnel & Decrypt and encrypt & $\mathrm{n}-1$ & Decrypted private key \\
& & $\mathrm{n}$ & Auth_n_pri Key \\
& & Public key \\
& & Auth_i_pub Key \\
\hline
\end{tabular}

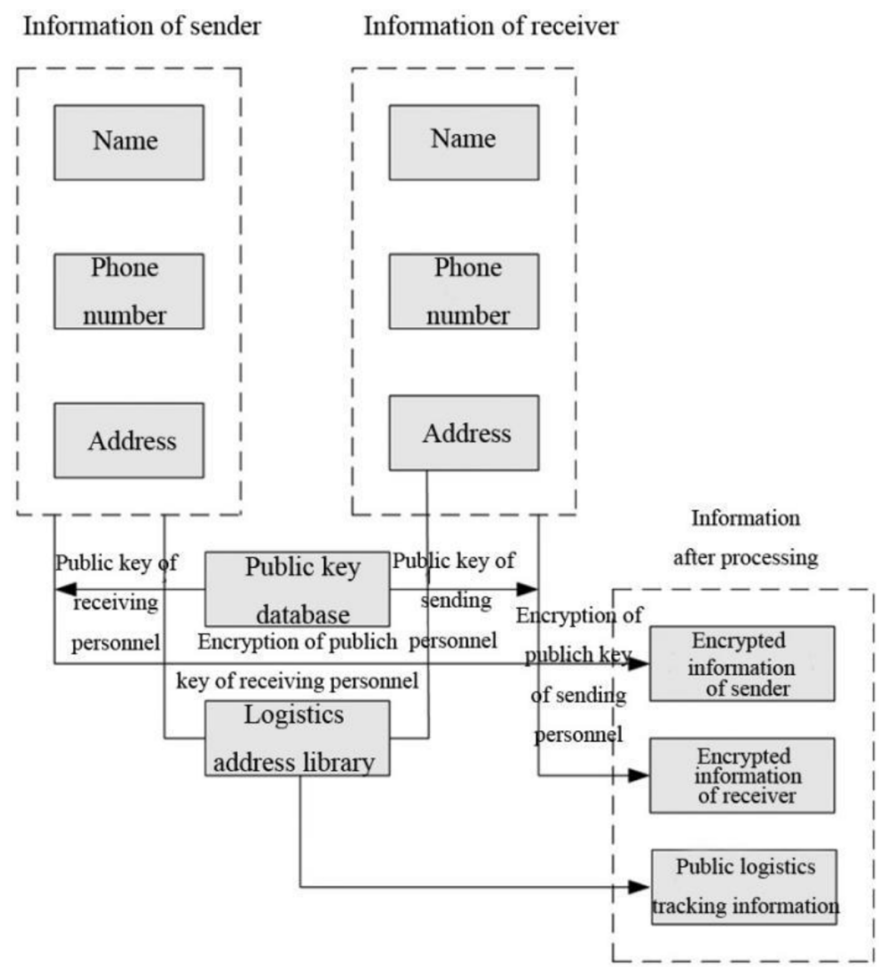

Fig. 3. Personal information encryption process.

\subsubsection{Personal information encryption}

In the e-commerce logistics process, the personal information of sellers and buyers mainly include name, telephone number and address. The process of encrypting information by using secrete key is shown in Figure 3. First, the receiver at the starting point made encryption treatment on the information of the sender using the public key, and then the information of the receiver is encrypted using the public key owned by the sending personnel at the terminal. As the receiving personnel is the starting point, it does not need to decrypt, and only encryption is needed, so the performance consumption of the terminal is not large; under such a situation, RSA encryption algorithm which is asymmetric can be used [11]. After calculation, all encrypted information is stored in the form of two-dimensional code.

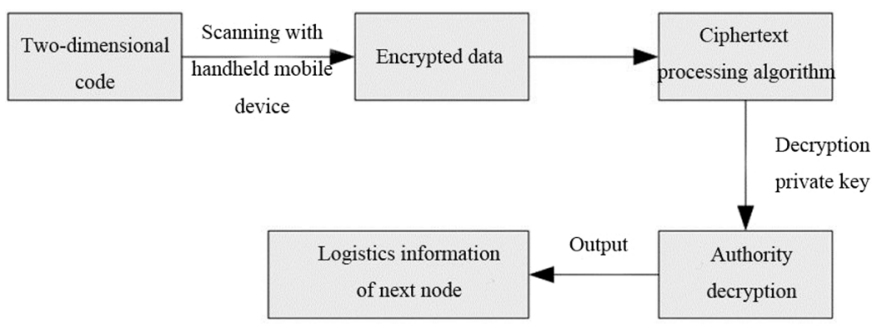

Fig. 4. Personal information decryption process.

\subsubsection{Decryption operation}

The scanning terminal of decryption operation contains the ciphertext processing algorithm, but according to the secrete key hierarchical distribution of logistics personnel, different personnel have different access to information. Staffs can get access to information after they input their code name (job number or account password) into the scanning terminal and the system checks it with the company system. Logistics staff scans two-dimensional codes with handheld terminals to obtain the corresponding knowable ciphertext information. The terminal decrypts the ciphertext using the decryption private key according to the ciphertext processing algorithm, so as to obtain the distribution conditions of the next node. The decryption process of personal information is shown in Figure 4.

\subsection{Anti-channel conflict scheme based on two-dimensional code}

\subsubsection{Design of product warehousing process}

Because of the huge volume of logistics orders, the variety of e-commerce products and the complexity of product information, for e-commerce logistics enterprises, antichanneling of goods when warehousing products [12] has become a very important part. Anti-channeling is to prevent the confusion of various items in the packaging line, so that the receiver receives the wrong goods. Twodimensional codes are graded and allocated to different grades of product packaging. By scanning the twodimensional codes on the express box, the storage information can be obtained. Figure 5 is a warehousing process of anti-channeling based on two-dimensional code.

First, the warehouse personnel input the information into the warehouse, and the corresponding two-dimensional code 


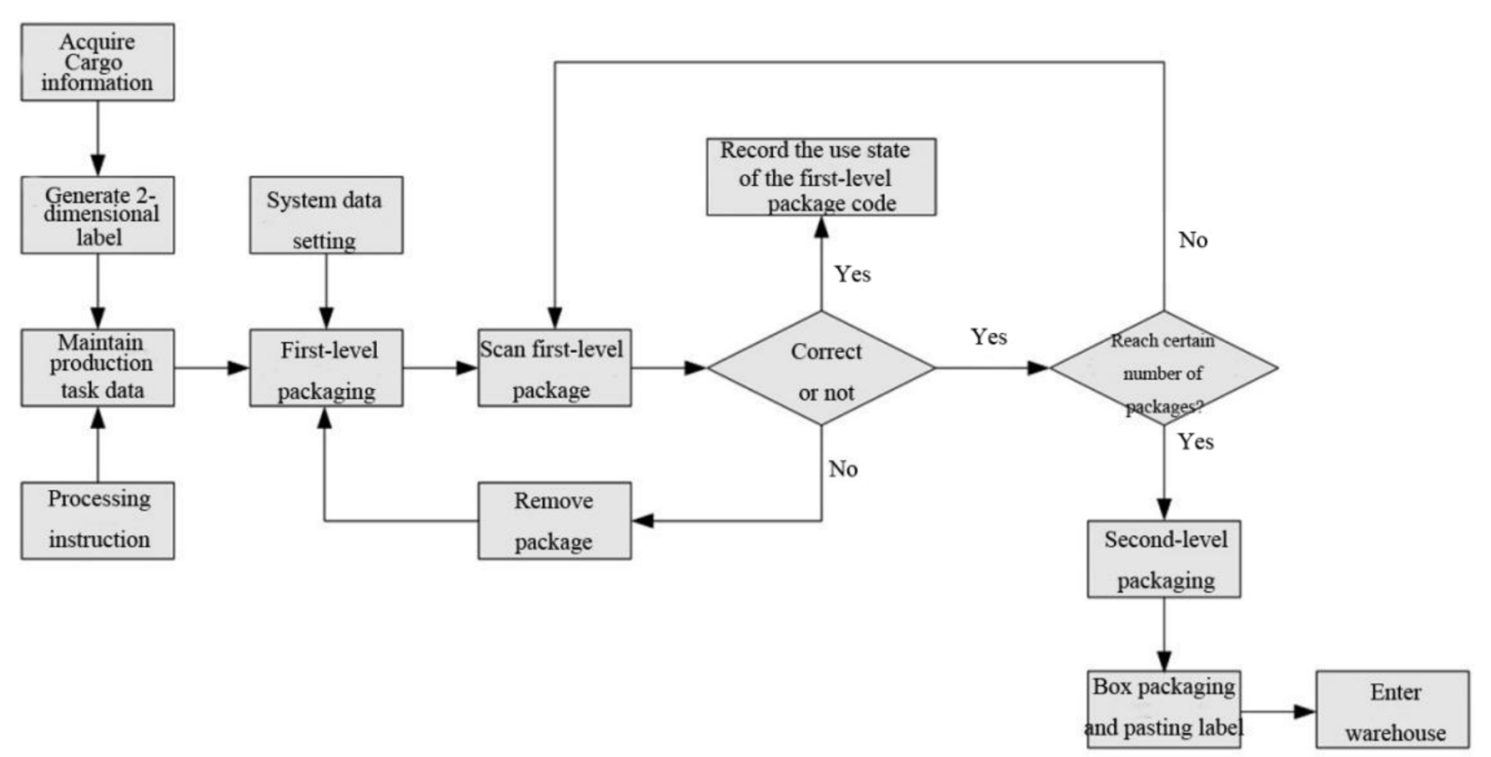

Fig. 5. Process of anti-channeling warehousing.

label is automatically generated by the code-spurting machine. Moreover, the first-level bagging packaging is carried out. When the goods arrive at the reading position, the two-dimensional code on the first-level package is scanned and identified by the reading equipment. If the recognition result is correct, the barcode status of the package is recorded and the goods are counted, otherwise the package is eliminated. If the number of packages reaches a certain standard, the second-level packaging, box packaging, will be carried out. The packaging code will be pasted and the boxes will be sealed for storage.

After storage, the packaging data will be uploaded to the management platform synchronously. In this way, every link of packaging can be queried, and the results can be compared with the actual data to find out if there is any channeling. The error information can be screened in detail by taking samples from the spot where there is channel conflict.

\subsubsection{Design of product warehousing module}

Warehousing module is the core of warehousing process. The warehousing module includes two subsystems: the code scanning module and the confirmation module.

\subsubsection{Code scanning module}

(1) Enter the express delivery number in the column of warehouse entry.

(2) Click on the "Lock" button. Set the entry column as read-only to prevent malicious changes; press the second time to unlock, and click on it to make it readable, that is, allow the outside world to change the entry number.

(3) Input box code.

(4) Click on "Complete code scanning" and enter the confirmation interface.

(5) The box number column shows the number of cargo boxes in real time. Box codes can be deleted manually after being selected.
(6) Exit the current interface and return to the home screen if the operator wants to abandon the operation of code scanning.

\subsubsection{Confirmation module}

(1) In the confirmation interface, the storage list and number of boxes are checked and counted.

(2) In the general warehouse, after receiving the information uploaded into the warehouse, it is encapsulated and sent to the server using the protocol, and exit the interface if the upload is successful.

(3) In the sub-warehouse, click the "Save" button to directly store the information as there is no network connection. When there is network connection, the warehousing information is re-entered.

(4) To cancel the confirmation operation, click the "Cancel" button.

\subsection{Logistics traceability scheme based on two- dimensional code}

With the improvement of the quality of life, consumers pay more attention to the quality of logistics products, especially the freshness of some fresh products. Therefore, traceability system is needed to achieve real-time monitoring and management of product quality.

\subsubsection{Design of traceability encoding}

In order to achieve product traceability, it is necessary to understand the relevant information in the process before the logistics process. In this study, factors such as producer, origin, date of production and product type are taken as the coding object, and traceability encoding is carried out according to the rules in GB/T 16986-2003, EAN - UCC System Application Identifier [13].

- Products are represented by trade item symbol AI (01), totaling 14 bits. 
Table 2. Calculating rules of check code.

\begin{tabular}{|c|c|c|c|c|c|c|c|c|c|c|c|c|c|c|}
\hline \multicolumn{15}{|c|}{ Digit position } \\
\hline & & & & & & N1 & $\mathrm{N} 2$ & N3 & $\mathrm{N} 4$ & N5 & N6 & N7 & $\mathrm{N} 8$ & $\mathrm{EAN} / \mathrm{UCC}-8$ \\
\hline & & N1 & $\mathrm{N} 2$ & N3 & $\mathrm{N} 4$ & N5 & N6 & N7 & N8 & N9 & N10 & N11 & N12 & UCC-12 \\
\hline & N1 & $\mathrm{N} 2$ & N3 & $\mathrm{N} 4$ & N5 & N6 & N7 & $\mathrm{N} 8$ & N9 & N10 & N11 & N12 & N13 & $\mathrm{EAN} / \mathrm{UCC}-13$ \\
\hline N1 & $\mathrm{N} 2$ & N3 & N4 & N5 & N6 & N7 & N8 & N9 & N10 & N11 & N12 & N13 & N14 & $\mathrm{EAN} / \mathrm{UCC}-14$ \\
\hline \multicolumn{15}{|c|}{ Multiply each position by the corresponding value } \\
\hline 3 & 1 & 3 & 1 & 3 & 1 & 3 & 1 & 3 & 1 & 3 & 1 & 3 & 1 & \\
\hline
\end{tabular}

Summation of product results

The sum result is subtracted from an number which is 10 times greater than or more than 10 times greater than the result value, and the result is the check code value.

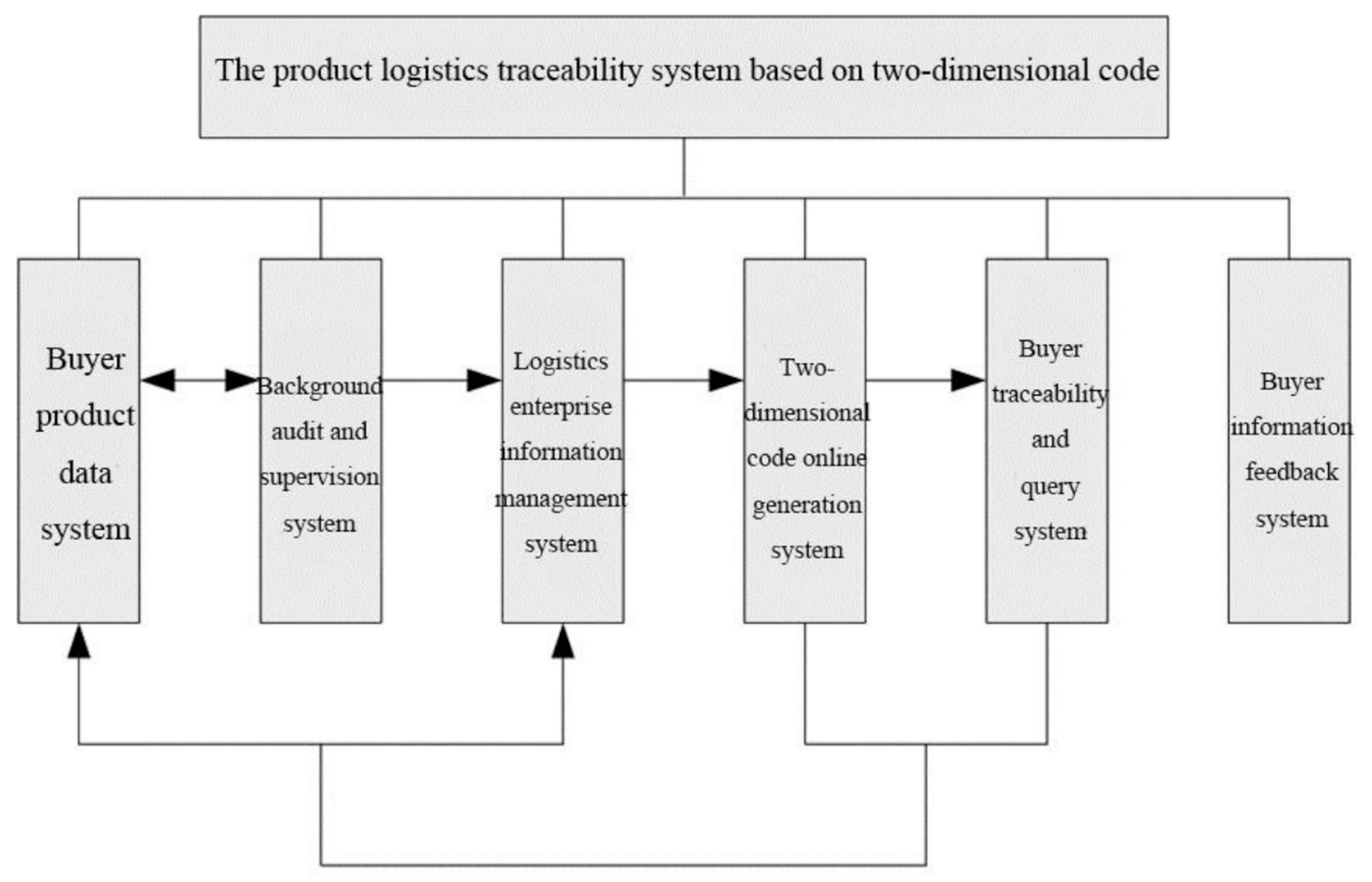

Fig. 6. Product logistics traceability system based on two-dimensional code.

- The production date is indicated by the production date symbol AI (11), which is 6 digits.

- The batch is represented by the packing date symbol AI (13), totaling 6 bits.

- Origin is represented by source entity reference symbol AI (251).

Using the above codes, the corresponding check codes can be calculated, and the two-dimensional code can be obtained from the check codes. The calculation rules of check codes [14] are shown in Table 2.

\subsubsection{Construction of traceability system}

According to the data uploaded by e-commerce sellers on the commodity details page and the actual operation of corresponding e-commerce logistics enterprises, a two- dimensional code traceability management system of product logistics composed of six subsystems can be constructed, as shown in Figure 6.

The main design of the system is as follows:

- In the background audit and supervision system, the administrator of logistics enterprise network background mainly checks the product information submitted by the merchant. If the material submitted by the merchant was consistent with the material submitted by the seller and conforms to the relevant national laws and regulations, the application will be passed.

- In order to ensure the stability of the traceability process, once the product information has been audited and approved, it will be automatically entered into the information management system of the logistics enterprise and cannot be modified. 
Table 3. Testing results of personal information encryption.

\begin{tabular}{|c|c|c|c|}
\hline Operation & $\begin{array}{l}\text { Scanning the two-dimensional } \\
\text { code on document by terminal (1) }\end{array}$ & $\begin{array}{l}\text { Scanning the two-dimensional } \\
\text { code on document by terminal (2) }\end{array}$ & $\begin{array}{l}\text { Scanning the two-dimensional } \\
\text { code on document by terminal (3) }\end{array}$ \\
\hline Result & $\begin{array}{l}\text { "Sender: Wang **" } \\
\text { "next station: site A" }\end{array}$ & $\begin{array}{l}\text { "Receiver: Zhao **" } \\
\text { "Address: No. 233, Y Street, } \\
\text { X City" } \\
\text { "Contact information: } \\
\text { 173XXXX5656" }\end{array}$ & $\begin{array}{l}\text { "ahogavioajgv'aj avlja'pv } \\
\text { aapjajfpajfpfpaaj" }\end{array}$ \\
\hline
\end{tabular}

- The logistics traceability system is designed according to PDCA (plan-do-check action cycle) closed-loop management structure [15], which adds the feedback board of buyer information. Buyers can submit suggestions for quality damage during logistics.

\section{Simulation analysis of two-dimensional code application}

\subsection{Testing environment}

In this study, the simulation test was carried out on the two-dimensional code based e-commerce logistics process in a laboratory. The information encryption, anti-channel conflict and traceability system based on two-dimensional code described above ran in the laboratory server. The relevant parameters of the server included quad-core i7CPU, $16 \mathrm{G}$ memory and $1024 \mathrm{G}$ hard disk. The server was divided into three virtual machines to run the above three systems. The parameters of each virtual machine were dual core CPU, 4G memory and $40 \mathrm{G}$ hard disk.

\subsection{Testing program}

\section{(1) Personal information encryption}

Firstly, an express document containing the name, address and contact information of the sender and receiver and the address of the transfer station was written; then the information in the express document was input into the information encryption system; the information encryption system encrypted the express information and printed out the a two-dimensional code, as shown in Figure 7. The plaintext in Figure 7 was used to display the express information, which was not displayed in the simulation experiment, and only the two-dimensional code was displayed.

Then three two-dimensional code scanning terminals were used to scan the two-dimensional code, and the scanning results were displayed in the computer. Terminals (1) and (2) were connected with the logistics encryption system, and they had the authority of transfer station and sender respectively. Terminal (3) was not connected with the logistics encryption system, and they did not have any authority to view the logistics information.

\section{(2) Cargo anti-channel conflict}

Firstly, part of the laboratory space was divided into three areas, a, b and c, and each of them was equipped with a

\author{
Sender: Mingming Wang \\ Receiver: Mingming Zhao \\ Address of receiver: No. 233, \\ Y Street, Y City \\ Contact: 173XXXX5656
}

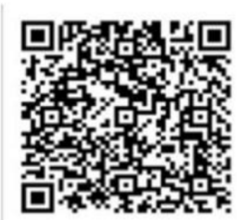

Fig. 7. Express document containing a two-dimensional code for simulation test.

two-dimensional code scanning terminal. The terminal was connected with the anti-channeling system to obtain the corresponding area number to indicate the area that the scanning terminal belonged to. Then the two-dimensional code generated in the last test was input into the system, and the system decrypted the two-dimensional code with the assigned key, then input area in which the cargo should be allocated, and then re-encrypted to generate the twodimensional code. The regenerated two-dimensional code was pasted on the outside of the carton and placed in three areas, a, b and c, respectively to record the scanning results of the terminal.

\section{(3) Logistics tracing}

The logistics route was set: site A-site B-site C. As the experiment was a simulation experiment, three scanning terminals were taken as the cargo logistics recording devices because of the limited space. The two-dimensional code generated according to the express document in (1) was tested; in addition to the basic express information, the address of logging logistics traceability system was also included. Then the carton which was pasted with the twodimensional code was scanned using three scanning terminals from site $\mathrm{A}, \mathrm{B}$ and $\mathrm{C}$ twice to represent warehousing and ex-warehousing. Finally the two-dimensional code was scanned by the handheld two-dimensional code scanning terminal to login the traceability system and query logistics track.

\subsection{Testing results}

The test results of personal information encryption are shown in Table 3 . The scanning result of terminal (1) which had the authority of transfer station only displayed the 
Table 4. Testing results of anti-channeling.

\begin{tabular}{llll}
\hline Operation & $\begin{array}{l}\text { Scanning the two-dimensional } \\
\text { code pasted on the carton using } \\
\text { the terminal of area a }\end{array}$ & $\begin{array}{l}\text { Scanning the two-dimensional } \\
\text { code pasted on the carton using } \\
\text { the terminal of area b }\end{array}$ & $\begin{array}{l}\text { Scanning the two-dimensional } \\
\text { code pasted on the carton using } \\
\text { the terminal of area c }\end{array}$ \\
\hline Result & $\begin{array}{l}\text { "The goods are in the right } \\
\text { warehouse and please continue } \\
\text { the step of warehouse out" }\end{array}$ & $\begin{array}{l}\text { "The goods are in the wrong } \\
\text { warehouse and please place the } \\
\text { goods in the right warehouse, and } \\
\text { the correct position of the cargo } \\
\text { is area a" }\end{array}$ & $\begin{array}{l}\text { "The goods are in the wrong } \\
\text { the correct position of the cargo } \\
\text { is area a" }\end{array}$ \\
\hline
\end{tabular}

Table 5. Testing result of logistics traceability.

\begin{tabular}{llll}
\hline Location of cargo & Time of going into warehouse & Time of getting out of warehouse & Complexity of cargo \\
\hline A & $9: 00 \mathrm{AM}$ & $9: 05 \mathrm{AM}$ & Complete \\
B & $9: 10 \mathrm{AM}$ & $9: 15 \mathrm{AM}$ & Complete \\
C & $9: 20 \mathrm{AM}$ & $9: 25 \mathrm{AM}$ & Complete \\
\hline
\end{tabular}

surname of the sender and the location of the next logistics station, and the rest of the express delivery information was not shown. The scanning result of terminal (2) which had the authority of deliveryman only the surname, address and contact information of the receiver, and the rest of the express delivery information was not displayed. The scanning result of terminal (3) which was not connected with the system and did not have the right of viewing information did not display any effective information, but displayed a row of messy code. It was found from the scanning results of the three terminals that the logistics mode based on the two-dimensional code had an effective personal information protection effect, and the specific performance is shown in Figure 7. Only the scanning terminal with the right of viewing the logistics information could obtain the logistics information from the two-dimensional code, and the different rights could only obtain the logistics information of different parts, thus protecting the personal information to the maximum extent.

The testing results of the anti-channeling system in the logistics mode based on the two-dimensional code are shown in Table 4 . The terminal scanning result of area a was "the goods are in the right warehouse and please continue the step of warehouse out". The terminal scan result of area b and c was "the goods are in the wrong warehouse and please place the goods in the right warehouse". It was seen from Table 4 that the terminals in the three areas could effectively identify the twodimensional code on the carton, obtain the cargo placement area contained therein, and compare it with the area where the terminal was located. If the set placement area of cargo was consistent with the area where the terminal was located, then the next step of warehouse out arrangement continued; if not, the cargo placement error prompted and the step of warehouse out stopped. Using two-dimensional code can effectively identify the goods misplaced in the warehouse, and using the scanning terminal can scan multiple two-dimensional codes at the same time, which greatly reduces the human consumption, prevents channeling to the largest extent, and ensures customers to receive the correct cargo.

In the simulation experiment, the two-dimensional code which passed the scanning of terminals from site A, B and $\mathrm{C}$ was equivalent to completing the logistics program of route A-B-C, and the carbon which carried the twodimensional code was equivalent to completing logistics in the simulated scenario. The logistics track of the carton which carried the two-dimensional code can be acquired from the logistics traceability system. Table 5 shows that the cargo passed through site A, B and C and it also shows the arrival and leaving time and complexity of the cargo. The logistics track displayed by the scanning results was consistent with the set sequence in the simulation of logistics track, indicating that the two-dimensional code based logistics mode could effectively record the logistics track of cargo and moreover the passing site, in-out time and complexity of the cargo can be queried through the two-dimensional code.

\section{Conclusion}

This study took optimizing the operation mode of ecommerce logistics as the goal, respectively analyzed personal information of users, packaging anti-channeling system and product traceability in the process of ecommerce logistics, and carried out simulation experiment on the two-dimensional code based personal information encryption system, anti-channeling system and logistics traceability system in the laboratory. In the simulated experiment of personal information encryption, the 
terminal without authority could only obtain messy code after scanning the two-dimensional code, and the terminal with authority could only obtain the information of the corresponding authority after scanning, but could not obtain all the information. In the simulated experiment of anti-channeling, terminals from different areas could determine whether a good was located in the correct area according to the two-dimensional code and make corresponding feedback. In the experiment of logistics traceability, scanning the two-dimensional code on the cargo could connect to the logistics traceability system and query the logistics track of cargo, and the queried result was the same with the actual logistics track.

Through the analysis and simulation experiment of the personnel information encryption, anti-channeling and logistics traceability in the two-dimensional code based logistics mode, this study verified that the logistics mode could effectively protect personnel information, prevent misplacement of cargo and query logistics track. The direction of future research is to further improve the encryption of personnel information by two-dimensional code and the performance of the two-dimensional code based anti-channeling and product traceability.

This study was supported by the Research Center for Ecommerce Entrepreneurship Innovation of Chongqing Youth Vocational \& Technical College (CQY2018CXTDC03).

\section{References}

1. H. Zhang et al., Comput. Optim. Appl. 72, 827-848 (2019)

2. Y. Shan et al., Proc. Second Int. Conf. Innov. Comput. Cloud Comput. 222-224 (2013)

3. X. Xiao, Z. Fu, Y. Zhang, Z. Peng, X. Zhang, J. Food Process Eng. 40, 1 (2015)

4. Y.J. Di, J.P. Shi, G.Y. Mao, Mod. Phys. Lett. 31, 1740035 (2017)

5. Y. Kalkan et al., Efficiency and Logistics 253-260 (2013)

6. X. Zhang et al., in 2016 IEEE Trustcom/BigDataSE/I SPA 996-1000 (2016)

7. S.F.W. Lim, X. Jin, J.S. Srai, Int. J. Phys. Distrib. Logist. Manag. 48, 308-332 (2018)

8. J.P. Yang, C.C. Yang, X.X. Yao, J. Bus. Econ. 16-22 (2014)

9. Y. Cheng, Z. Fu, B. Yu, IEEE Trans. Inf. Foren. Sec. 1-1 (2018)

10. R. Kulshreshtha, A. Kamboj, S. Singh, Int. Conf. Comput. Sci. $722-731$ (2012)

11. U. Somani, K. Lakhani, M. Mundra, Int. Conf. Parallel Distrib. Grid Comput. 211-216 (2010)

12. X.Q. Liu et al., J. Cent. South Univ. 42, 1890-1898 (2011)

13. J. Montero-Mayorga, C. Queral, J. Gonzalez-Cadelo, Ann. Nucl. Energy 75, 87-100 (2015)

14. D.J.C. Mackay, R.M. Neal, Electron. Lett. 32, 457-458 (2013)

15. K. Kenk, T. Haldma, Discuss Estonian Econ. Policy 24, $35-50(2016)$

Cite this article as: Jing Rao, Operation mode of electric business logistics based on the application of two-dimensional code technology, Int. J. Metrol. Qual. Eng. 11, 3 (2020) 